\title{
Kernos
}

Revue internationale et pluridisciplinaire de religion grecque antique

15 | 2002

Varia

\section{Lambros couloubARITSIS, Histoire de la philosophie ancienne et médiévale. Figures illustres}

\section{André Motte}

\section{OpenEdition \\ Journals}

Édition électronique

URL : http://journals.openedition.org/kernos/1461

DOI : 10.4000/kernos. 1461

ISSN : 2034-7871

\section{Éditeur}

Centre international d'étude de la religion grecque antique

Édition imprimée

Date de publication : 1 janvier 2002

ISSN : 0776-3824

\section{Référence électronique}

André Motte, "Lambros couloubaritsıs, Histoire de la philosophie ancienne et médiévale. Figures illustres », Kernos [En ligne], 15 | 2002, mis en ligne le 16 juin 2011, consulté le 24 septembre 2020 URL : http://journals.openedition.org/kernos/1461; DOI : https://doi.org/10.4000/kernos.1461 
Carthage, 248-258). Les liens qui unissent l'historiographie et la pastorale ou la théologie, sont étudiés chez M.-A. Calvet-Sébasti (L'évocation de l'affaire de Sasimes par Grégoire de Nazianze), E. Soler (L'utilisation de l'histoire de l'Église d'Antioche au $\mathrm{IV}^{\mathbf{e}}$ siècle par Jean Chrysostome, dans les débuts de sa prédication), et A. Le Boulluec (L'historiographie dans les écrits théologiques de l'empereur Justinien). Un cas spécial est celui de l'Église éthiopienne : à ce propos, voir J.-N. Pérès (Les origines du christianisme en Éthiopie : histoire, tradition et liturgie) et G. Lusini (L'Église axoumite et ses traditions historiographiques, $\mathrm{IV}^{\mathrm{e}}$-vII ${ }^{\mathrm{e}}$ siècle).

De ce qui précède, il ressort clairement que ce livre, comme beaucoup de recueils semblables, manque de cohérence. Il est regrettable aussi qu'il ne renferme pas de conclusions générales, dans lesquelles on aurait pu trouver la réponse à des questions fondamentales (par exemple : est-ce qu'on peut donner des éléments constitutifs qui valent pour la totalité de l'historiographie de l'Église ancienne? qui, parmi les historiographes chrétiens antiques, mérite vraiment le titre d'historien?, etc.).

Mais les qualités du livre ne peuvent pas être mises en doute. La plupart des articles sont importants et novateurs; ils sont écrits avec un soin et une exactitude remarquables; toujours, la littérature la plus récente a été assimilée. Les quelques

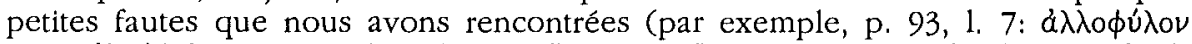

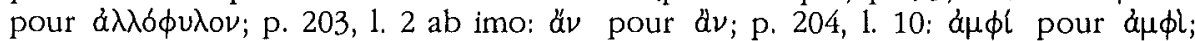
p. 363 n. 1: Antiochie pour Antiochië; p. 526, 1. 1: Chacédoine pour Chalcédoine), n'entament en rien la valeur de ce volume.

\section{Peter Van Deun \\ (Katholieke Universiteit Leuven)}

Lambros Couloubari'ssis, Histoire de la philosophie ancienne et médiévale. Figures illustres, Paris, Grasset, 1998. 1 vol. $15,5 \times 24,5 \mathrm{~cm}, 1326$ p. (Le Collège de Pbilosopbie). ISBN : 2-246-50991-2.

On devine qu'une somme aussi monumentale, propre à intéresser un large public, ne se lit pas en une soirée et que, pour en rendre compte, il faut bien laisser un peu de temps au temps... Mais il n'est pas trop tard pour la signaler à l'attention des lecteurs de Kernos. C'est que son A., bien connu comme historien de la philosophie, est aussi très intéréssé, comme on sait, par l'histoire des religions et par les questions relatives au mythe, un intérêt qui ne passe nullement ici inaperçu. Précisons tout d'abord que l'ouvrage ne fait pas double emploi avec celui qu'il a fait paraître, en 1992, sous le titre Aux origines de la philosophie européenne. De la pensée archaïque au néoplatonisme, Bruxelles, De Boeck, et qui a connu depuis lors deux rééditions (c.r. dans Kernos 7 [1994], p. 405-406). D'une part, cette nouvelle étude s'étend cette fois bien au-delà de l'Antiquité grécoromaine puisque elle couvre en outre toute la période médiévale et consacre même un ultime chapitre à la renaissance du platonisme, dont Pléthon de Mistra, au $x^{e}$ siècle, fut un des principaux initiateurs; comme on le voit, la philosophie byzantine, et aussi du reste la philosophie arabe, trouve une place, très originale, dans ce volume qu'habite un souci légitime de ne pas restreindre l'enquête au monde occidental latin. D'autre part, le renouvellement vient encore de ce que l'étude est ici davantage centrée sur les figures de proue qui jalonnent ces deux millénaires de réflexion philosophique et qu'elle s'attache à montrer comment et pourquoi ces figures ont été promues comme telles, parfois de façon très durable. Pareille visée implique que l'on prête une attention particulière aux contextes culturels des époques traversées. Dans une introduction substantielle, L'A. souligne d'ailleurs lui-même la nécessité de cette prise en compte : «Jamais la philosophie [ancienne et médiévale], écrit-il (p. 23), ne peut être analysée en dehors 
des démarches corrélatives qui concernent la sagesse, la science, la théurgie et la théosophie. Face à la puissance divine, les philosophes du passé ont eu beaucoup de difficulté à promouvoir une démarche qui soit uniquement et simplement philosophique ». Aussi voit-on, ajoute-t-il en substance, la thèse novatrice de l'bomme mesure de toutes choses que défendit un Protagoras héritier de tout un courant de sagesse, battue en brèche par Platon, pour qui c'est Dieu qui est la mesure de toutes choses, une position qui reste fidèle à la tradition archaïque et qu'avait consacrée déjà le pythagorisme. Or, en dépit des efforts consentis pour ébranler cette dernière conception (sceptiques et épicuriens) ou pour la modérer (aristotéliciens et stoïciens), c'est elle qui triomphera dans le monde romain, ouvert au pluralisme et tolérant à l'égard de la religiosité. Le courant pythagoricoplatonicien y sera bientôt renforcé par le syncrétisme religieux païen que l'époque verra naître, et aussi par le judaïsme mosaïque et le christianisme. Tout au long de son parcours, l'A. sera donc attentif à mettre en évidence ce facteur culturel, parmi d'autres qui ont aussi toute leur importance, comme les facteurs politiques.

Il convient d'attirer en particulier l'attention sur la première partie de l'ouvrage intitulée «Le temps de la production des mythes ». Fort de près de cent pages, ce chapitre introductif est consacré à une étude des généalogies hésiodiques et orphiques, mais aussi, - ce qui était moins attendu, mais fort bien venu pour faire apparaître des différences de perspective, - à un exposé des généalogies judaïque et judéo-chrétienne. Au préalable, on a droit à une réflexion de portée générale sur "Le mythe et la question de la distorsion ». L'A. y reprend d'abord des idées qu'il avait déjà eu l'occasion de développer précédemment (voir notamment Kernos 1 [1988], p. 111-120), comme l'importance d'une distinction à faire entre mythe et religion, le premier méritant dès lors d'être envisagé dans ses propres structures. Est aussi reprise et argumentée à nouveaux frais l'idée importante selon laquelle le mythe comporte un fondement rationnel et que «le discours rationnel lui-même ne saurait se développer en dehors de l'affectivité "; autrement dit, la philosophie n'est pas née d'un rejet du mythe au profit de la raison, il n'y a pas eu passage du mutbos au logos, mais passage d'un type de discours et de rationalité à un autre, mutation dans laquelle la diffusion de l'écriture a pu jouer un rôle majeur en renforçant la capacité mémorisatrice de l'homme et en stabilisant ainsi l'information. Dans la suite des temps, la civilisation occidentale, en dépit de l'illusion qu'elle a pu parfois nourrir d'un monde fondé uniquement sur la raison, ne s'est jamais coupée des pratiques antiques et chrétiennes du mythe.

L'A. souligne aussi à nouveau l'importance, dans la pensée archaïque grecque, du schème régulateur de la parenté qui permet d'unifier l'expérience humaine et de rendre intelligible sa relation au monde. Ce schème, que la philosophie conservera ( $c f$. la notion de genos), en y ajoutant d'autres, comme celui du chemin ou celui de l'artisan, assure la mémoire du passé et est lié à l'émergence d'un discours qui glorifie certaines figures ancestrales. Et de montrer comment la glorification relative à la mort a connu une envergure extraordinaire dans le cas de trois figures particulièrement emblématiques : Achille, Socrate et Jésus-Christ. Examinant ensuite les pratiques du mythe chez Platon, à bien des égards exemplaires, l'A. en vient à cette notion de distorsion qu'annonçait le titre de la section. Il faut entendre par là le décalage qui existe forcément entre la littéralité de la structure narrative et la réalité dont elle entend rendre compte, décalage que le philosophe prend parfois soin lui-même de souligner dans le cas des mythes eschatologiques, ce qu'il n'a pas fait cependant pour le récit de la création du monde dans le Timée, avec pour conséquence le fait que les uns ont pris le mythe à la lettre et que les autres, - essentiellement ceux qui ont adhéré à 
son école, - affirment qu'il s'agit d'un mythe pédagogique décrivant les structures fondamentales d'un monde éternel. Le récit mythique a donc toujours besoin d'être redressé, comme le soulignera explicitement Plotin; encore faut-il connaître les règles adéquates de ce redressement. Or la reconnaissance de ce genre de distorsion dans la pratique du mythe n'est pas une exclusivité des philosophes, mais remonte bien haut dans la littérature grecque, puisque, dans son prologue de la Théogonie, Hésiode fait dire aux Muses qui l'inspirent qu'elles sont capables de dire des mensonges semblables à la réalité, mais savent aussi, quand elles le veulent, proclamer des vérités. Autant dire que les mythes que le poète va produire ne sont pas à prendre pour argent comptant, mais doivent être redressés par la pensée. L'A. se livre à une exégèse intéressante de ce passage difficile et montre que, depuis cette époque jusqu'à la fin du Moyen Âge, en particulier chez Pléthon, les philosophes ne cesseront de faire effort pour surmonter les distorsions quasi nécessaires qui sont inhérentes au discours mythique, mais que les poètes quant à eux ne cherchent pas à élucider, sauf cas exceptionnels comme celui d'Hésiode.

Tout le déroulement de cette longue histoire de la pensée occidentale depuis ses origines jusqu'à la Renaissance est à l'avenant : rempli d'aperçus neufs et d'éclairantes synthèses témoignant d'une remarquable maîtrise.

A. Motte

(Université de Liège)

Marcel Detienne, Comparer l'incomparable, Paris, Seuil, 2000. 1 vol. $14 \times$ $20,5 \mathrm{~cm}, 140$ p. (La Librairie du XX $X^{e}$ siècle). ISBN : 2-02-036139-6.

De l'aveu même de l'A., ce livre est un pamphlet, et théorique, insiste-t-il. A la lecture du titre, on devine aisément contre qui la hache de guerre est ici déterrée. N'est-ce pas la science historique naissante qui, au siècle dernier, a fait son credo du dicton « on ne peut comparer que ce qui est comparable » et qui, à coup de jugements de valeur implicites, s'est arrogé le droit d'indiquer ce qu'il était permis de comparer ? Or on sait les dérives qu'a rapidement entraînées pareil préjugé : après 1870 , la science en question va se faire "nationale » et " des historiens en uniforme vont édifier la place forte de l'Incomparable ", des deux côtés du Rhin, s'employant à « forger l'identité d'une race et de ses forces de terre et de sang ». Et l'Angleterre à son tour sera bientôt atteinte de la même "acné de la Nation ». Une telle mentalité ne pouvait nourrir qu'indifférence superbe à l'égard des sociétés réputées sans civilisation, et $s i$, d'aventure, il se trouvait quelques nomades comparatistes, "ils étaient abattus à vue, sans sommation ". Seules pré vaudront désormais les valeurs occidentales, lesquelles pourront du moins, sous la pression des Nationaux, se réclamer d' " une transmission en ligne directe de l'universalité grecque ». C'est ainsi que, pour longtemps, "La Grèce, décrétée éternelle, est mise sous cloche ", que les hellénistes multiplient les panneaux d'interdiction et que "le Patrimoine de l'Humanité est confié à des gardiens sûrs, triés sur le volet : les hellénistes académiciens. Momification garantie. ». Même aujourd'hui, tous les "barbelés des disciplines universitaires" sont loin d'avoir cédé, les historiens demeurant "pris au piège d'une culture qui se referme comme une huitre ». Et il se trouve toujours des académiciens pour dénoncer " la fâcheuse collision de la Grèce avec la chose comparative ", les Grecs, c'est bien connu, n'étant pas comme les autres. «Respect des douaniers et passeport bien en règle » vous garantiront donc honneurs et décorations.

Quelle régression! Car il y eut, si l'on ose dire, un âge d'or, durant lequel on pouvait faire converser librement les anciens Grecs avec les tribus du Nouveau Monde, et il y eut aussi le temps des Fontenelle et autres Lafitau qui continuèrent 